\title{
Cross-Modal Pattern of Brain Activations Associated With the Processing of Self- and Significant Other's Name
}

\author{
Pawel Tacikowski, '* André Brechmann, ${ }^{2}$ and Anna Nowicka' \\ ${ }^{1}$ Nencki Institute of Experimental Biology, Department of Neurophysiology, Laboratory of \\ Psychophysiology, 02-093 Warsaw, Poland \\ ${ }^{2}$ Leibniz-Institute for Neurobiology, Special-Lab Non-Invasive Brain Imaging, 39118 Magdeburg, \\ Germany
}

\begin{abstract}
Previous neuroimaging studies have shown that the patterns of brain activity during the processing of personally relevant names (e.g., own name, friend's name, partner's name, etc.) and the names of famous people (e.g., celebrities) are different. However, it is not known how the activity in this network is influenced by the modality of the presented stimuli. In this fMRI study, we investigated the pattern of brain activations during the recognition of aurally and visually presented full names of the subject, a significant other, a famous person and unknown individuals. In both modalities, we found that the processing of self-name and the significant other's name was associated with increased activation in the medial prefrontal cortex (MPFC). Acoustic presentations of these names also activated bilateral inferior frontal gyri (IFG). This pattern of results supports the role of MPFC in the processing of personally relevant information, irrespective of their modality. Hum Brain Mapp 34:2069-2077, 2013. @ 2012 Wiley Periodicals, Inc.
\end{abstract}

Key words: self-name; self-recognition; self; cortical midline structures; medial prefrontal cortex; fMRI

\section{INTRODUCTION}

There are many ways in which social communication can be initiated and different sensory channels are used for this purpose [Kampe et al., 2003]. The most obvious way is to call the name of a person we would like to com-

Contract grant sponsor: Polish Ministry of Science and Higher Education; Contract grant number: IP2010043270; Contract grant sponsor: European Union; Contract grant number: 1211080013.

*Correspondence to: Pawel Tacikowski, Nencki Institute of Experimental Biology, Department of Neurophysiology, Laboratory of Psychophysiology, 3 Pasteur Street, 02-093 Warsaw, Poland. E-mail: p.tacikowski@nencki.gov.pl

Received for publication 28 February 2011; Revised 3 December 2011; Accepted 2 January 2012

DOI: $10.1002 / \mathrm{hbm} .22048$

Published online 19 March 2012 in Wiley Online Library (wileyonlinelibrary.com). municate with. Our own name, presumably due to its high social value, seems to have preferential status in information processing, i.e., its detection is highly automatic [e.g., Cherry, 1953; Gronau et al., 2003; Moray, 1959; Shapiro et al., 1997]. Moreover, self-name seems to trigger some self-referential processes, as suggested by the "name-letter effect" [Wentura et al., 2001; see Koole and Pelham, 2003 for review]. Interestingly, children who are later diagnosed with autism spectrum disorder (ASD) show a delayed or even a lack of reaction to self-name ("Does your child respond to his/her name when you call?" is one of the critical items in the CHAT screening tool for ASD) [Baron-Cohen et al., 1992]. From about 5 months old, healthy infants are not only able to recognize their name, they also use it as a social cue to guide their attention to events and objects in the external world [Parise et al., 2010].

These observations have instigated a number of studies aimed at identifying neural correlates of such preference 
in self-name processing. Studies employing fMRI and PET have most consistently demonstrated increased activation of the medial prefrontal cortex (MPFC) during self-name processing [Carmody and Lewis, 2006; Holeckova et al., 2008; Kampe et al., 2003; Perrin et al., 2005; Staffen et al., 2006]. The role of the bilateral inferior frontal gyri, superior temporal gyrus, and precuneus was also emphasized. It is noteworthy that in all of these studies, self-name-specific activations were identified by comparing the processing of self-name with that of unknown names. This might be problematic, since unknown names differ not only in terms of the "me vs. not-me" distinction, but also with regard to their significance to the subject ("very important to me" vs. "not very important to $\mathrm{me}^{\prime \prime}$ ), as well as their general familiarity (one most probably hears his or her own name more often than any other name). As a consequence, it is hard to determine whether the effects observed in these previous studies were due to self-specific processing per se [e.g., see Laureys et al., 2007 for review]. In addition, all of the aforementioned studies used only auditory presentations of names, which prevented the identification of the more general (i.e., crossmodal) pattern of activation associated with self-related information processing.

A more recent study by Sugiura et al. [2008a] extended the set of control conditions by introducing a friend's name (closer to self-name in terms of emotional salience and familiarity) and used visual presentations of names. Interestingly, when the processing of self-name was directly compared with the processing of a friend's name, no significant activations were found. Instead, common activations for self- and a friend's name were detected in the cortical midline structures (CMS), i.e., MPFC and in the anterior and posterior cingulate cortices (ACC and PCC). These results suggested that the involvement of cortical midline structures-MPFC in particular-is determined more by the personal relevance of a name than by the fact that it designates a subject (i.e., self-specificity). We would like to emphasize that in this context, we use "personal-relevance" (or self-relatedness) as a more general term than "self-specificity," i.e., both self- and close other's name are personally relevant, whereas only self-name is self-specific.

This study was designed to test whether the activation of the CMS during name recognition is associated with self-specificity or personal relevance. A novel aspect of the study was the presentation of the names in two modalities, which enabled the investigation of cross-modal and modality-specific patterns of brain activations. As a consequence, the first aim of this study was to identify brain structures which show self-specific activations that are common to or particular for the auditory and visual modalities. To achieve this we compared the processing of self-name vs. close other's name. The second aim was to investigate brain regions showing increased neural responses to personally relevant names (i.e., both self- and close other's name), again identifying the structures acti- vated in one or both of the modalities. To obtain this goal we compared the processing of self- and close other's name with the processing of a less personally relevant name, i.e., a famous person's name. In addition, we were interested whether effects of self-specificity and/or personal-relevance could be explained by more general effects, such as general familiarity [e.g., see Laureys et al., 2007; Qin et al., 2012]. To test this possibility we searched for an overlap in brain activations for self-specificity/personalrelevance and general familiarity (general familiarity was assessed in the famous > unknown name contrast).

We investigated blood-oxygen-level-dependent (BOLD) responses during the processing of full names (still called "names" for ease of reference), presented visually or aurally (there were two separate sessions in which a given type of stimulus was presented). The task was a simple familiar vs. unfamiliar discrimination. Thus, a total of 12 conditions comprised a two-factorial design composed of factor modality (two levels) and type of name (six levels). The data recorded during the aural part of the experiment have already been used to investigate another research question [Tacikowski et al., 2011].

\section{METHODS}

\section{Participants}

Twenty-four right-handed subjects (12 females, 12 males, mean age: $25.3 \pm 3.6$ years) participated in this study. None of them had ever changed their first or last names. All subjects gave their written informed consent to the study, which was approved by the Ethical Committee of the University of Magdeburg (in compliance with the Code of Ethics of the World Medical Association-Declaration of Helsinki). In both parts of the experiment two subjects were excluded from further analyses due to errors in their sets of stimuli. In the visual part, one subject was excluded due to excessive head movement. As a consequence, data recorded for a total of 21 subjects (the same in both parts) were included in the analyses.

\section{Stimuli and Task}

First and last names were presented visually (v) or aurally (a) and belonged to six categories: (1) the subject's own name-Sa, Sv; (2) a significant other's name-Sia, Siv; (3) a famous person's name-Fa, Fv; (4) an unknown person's name sharing the first name of the subject-USa, USv; (5) an unknown person's name sharing the first name of the significant other-USia, USiv; and (6) an unknown person's name sharing the first name of the famous person-UFa, UFv. Using three unfamiliar names with the same first names as self, significant other and a famous person was supposed to increase the difficulty of the task and maintain the subjects' attention, because the first names or even the first letters of the first names could 
not be additional cues. The task was a simple familiar vs. unfamiliar discrimination. The participants pressed one button for familiar names and another button for unknown names on a two-button MRI-compatible response pad, using the right index and middle fingers to indicate their responses. Because the type of behavioral response for "self," "significant other," and "famous" conditions was the same, this factor did not confound the possible differences between these conditions.

The subjects were told that the accuracy of their responses was more important than speed (this manipulation was to reduce the error rate). A few weeks before the experiment the participants were asked to identify a person who was most significant in their life (4 subjects gave the names of their mothers; 7 , best friends; 7 , boyfriends or girlfriends; 3 , wives; 3 , brothers or sisters). The other names in each six-name set were chosen individually for each subject so as to control for the gender ( 3 female and 3 male names in each set) and the length of the names. In the "famous name" category, 24 different names were selected (12 well-known female and 12 well-known male athletes and entertainers), but each subject saw/heard only one of them (there were 24 subjects and each saw/ heard a different famous name). As a consequence, each subject had a different, individually tailored, set of stimuli in which the number of repetitions of a particular stimulus was equal across conditions. All of the names were of German origin (to match the subjects' own and significant others' names). Before the experiment, each subject was asked to confirm that he or she knew the famous name and did not know the unknown names in his or her set of stimuli. To minimize habituation and to mimic real-life situations (in their everyday life people hear different names spoken by different people and read different names written in different fonts), each name in a set was read by six different speakers or written in six different fonts. Three male and three female voices were used. The recordings were performed by professional actors and the same actors read all the names for all participants. As a result, the effect of voice was fully counterbalanced across subjects and across conditions. Similarly, all the names were written in the same six fonts and the same six fonts were used for all the participants.

The size of the visual stimuli ranged from $4 \times 6$ to $4 \times$ 8 visual degrees. The length of the stimuli did not differ significantly between categories (the number of letters \pm standard deviation: $\mathrm{Sv}-12 \pm 2$; Siv-12 \pm 2 ; Fv-12 \pm 2; USv-13 \pm 2 ; USiv-13 \pm 2 ; UFv-12 \pm 2 ). Similarly, the length of the auditory stimuli did not differ significantly between categories (time in seconds \pm standard deviation: $\mathrm{Sa}-1.2 \pm 0.1 ; \mathrm{Sia}-1.2 \pm 0.2 ; \mathrm{Fa}-1.1 \pm 0.1 ; \mathrm{USa}-1.2 \pm$ 0.1 ; USia-1.1 $\pm 0.1 ; \mathrm{UFa}-1.1 \pm 0.1$ ). The peak volume of each auditory stimulus was normalized using Adobe Audition $₫$ software (version 3.0, Adobe Systems Incorporated, San Jose, CA).

In the two parts of the experiment employing the visual and auditory modalities, stimuli from each category were presented 18 times in pseudorandom order (not more than three successive presentations of the same type of name or the same font/voice). Each part of the experiment consisted of 108 trials. The order in which the two parts were carried out was counterbalanced: half of the subjects were assigned to the visual name-recognition task first while the other half were asked to begin with auditory name-recognition. The pause between these two parts was about 5 min. Each trial began with a "fixation" point (a white cross or a "beep" sound presented for $500 \mathrm{~ms}$ ), followed by the presentation of the target name. Visual stimuli were displayed for 1,000 ms, whereas the time of presentation for the auditory stimuli corresponded to the recording duration. Next, the subjects had time to respond (no stimulus presented). Each trial lasted for $7.5 \mathrm{~s}$ in total. The intertrial interval (ITI) ranged from 1 to $3 \mathrm{~s}$. This temporal jitter was included to optimize the blood-oxygen-level-dependent (BOLD) response and signal discrimination between stimulus categories [Wager and Nichols, 2003]. The software package PRESENTATION (Neurobehavioral Systems, Albany, CA) was used to present stimuli and record responses.

\section{Behavioral Data Analysis}

The accuracy rate and reaction times (RTs) were analyzed using two-way repeated measures ANOVA, where modality (auditory vs. visual) and type of name (S, Si, F, US, USi, UF) were the factors. Violations of sphericity were adjusted according to the Greenhouse and Geisser [1959] formula. In the post hoc analysis, the Bonferroni correction for multiple comparisons was used.

\section{fMRI Data Acquisition}

A 3 Tesla scanner (Siemens Trio, Erlangen, Germany), equipped with an eight-channel head coil, was used to collect fMRI data. Prior to the study phase, detailed anatomical data (256 slices) of the whole brain were obtained using a multiplanar rapidly acquired gradient echo (MPRAGE) sequence with 1-mm isotropic resolution. In the study phase, functional volumes were collected using echo planar imaging $(\mathrm{EPI})(\mathrm{TE}=30 \mathrm{~ms}$; $\mathrm{TR}=2 \mathrm{~s}$; $\mathrm{FA}=$ $80^{\circ}$, slice thickness $3.5 \mathrm{~mm}$; matrix size $64 \times 64 ; 3.5-\mathrm{mm}$ isotropic resolution). Every 2 s, 34 contiguous, obliqueaxial images were obtained with the total of 543 brain volumes.

\section{fMRI Data Analysis}

The imaging data were analyzed using Statistical Parametric Mapping software (SPM8, Wellcome Department of Cognitive Neurology, London, UK). First, functional images were motion and slice-time corrected. Then, structural images from single subjects were coregistered to the mean functional image. Next, the unified normalization 
Tacikowski et al.

TABLE I. Behavioral data

\begin{tabular}{|c|c|c|c|c|c|c|c|}
\hline & & S & $\mathrm{Si}$ & F & US & USi & UF \\
\hline \multirow[t]{2}{*}{ Correct responses (\%) } & A & $97 \pm 1.2$ & $97 \pm 1.5$ & $97 \pm 1.3$ & $98 \pm 1.2$ & $94 \pm 2.6$ & $95 \pm 1.6$ \\
\hline & $\mathrm{V}$ & $99 \pm 0.4$ & $99 \pm 0.4$ & $99 \pm 0.6$ & $98 \pm 0.8$ & $99 \pm 0.5$ & $98 \pm 0.6$ \\
\hline \multirow[t]{2}{*}{ Mean RT (ms) } & A & $1671 \pm 59$ & $1692 \pm 58$ & $1570 \pm 70$ & $1727 \pm 64$ & $1734 \pm 53$ & $1684 \pm 60$ \\
\hline & $\mathrm{V}$ & $1421 \pm 60$ & $1432 \pm 57$ & $1461 \pm 62$ & $1510 \pm 59$ & $1487 \pm 57$ & $1525 \pm 59$ \\
\hline
\end{tabular}

The percentage of correct responses and the mean reaction times (RT) in milliseconds for each condition in the auditory (A) and visual (V) parts of the experiment. Values are the mean \pm standard error of the mean. S, self-name; Si, significant other's name; F, famous person's name; US, unknown name with the same first name as the subject; USi, unknown name with the same first name as the significant other; UF, unknown name with the same first name as the famous person.

routine was conducted with voxel size $2 \times 2 \times 2 \mathrm{~mm}$ (as a template we used the Montreal Neurological InstituteMNI T1-averaged image). Finally, the data were smoothed using an 8-mm Gaussian FWHM algorithm in 3D.

For each subject, the onset and duration of each stimulus were modeled in a general linear model, according to the distinct stimulus types. Regressors were convolved with the canonical hemodynamic response function, separately for the auditory and visual parts of the study. Specific condition effects were assessed by the application of linear contrasts, where parameter estimates for the events were compared to the "global" (e.g., 1000000 for Sa). This resulted in $t$-statistics for each voxel. For the group analysis, the contrast images created for each subject were entered into a two-way ANOVA (modality $\times$ type of name), constituting a random-effects model [Friston et al., 1995].

We tested the cross-modal self-specific activations by conjunction analysis (i.e., Sa-Sia conj. Sv-Siv). To extract self-name-specific activation for the auditory modality alone, a contrast for the interaction [i.e., (Sa-Sia)-(Sv-Siv)] was computed and masked inclusively by Sa-Sia to ensure that the activation was Sa-specific, rather than Siv-specific. Analogously, to extract self-name-specific activation in the visual domain alone, a contrast for the interaction [i.e., (Sv-Siv)-(Sa-Sia)] was masked inclusively by Sv-Siv.

The cross-modal pattern of brain activations for personally relevant names was also identified by conjunction analysis (i.e., Sa-Fa conj. Sia-Fa conj. Sv-Fv conj. Siv-Fv). To extract modality-specific activations for self- and closeother's name we computed the following interactions: (1) (Sa-Fa)-(Sv-Fv) masked inclusively by Sa-Fa; (2) (Sia-Fa)(Siv-Fv) masked inclusively by Sia-Fa; (3) (Sv-Fv)-(Sa-Fa) masked inclusively by Sv-Fv; and (4) (Siv-Fv)-(Sia-Fa) masked inclusively by Siv-Fv. Next, in order to reveal modality-specific activations common for personally relevant names we used conjunction analysis, i.e., auditoryspecific-conjunction of contrasts (1) and (2); visually specific - conjunction of contrasts (3) and (4).

Activations related to the general familiarity factor were assessed by comparing the famous names processing with the unknown names processing. These contrasts were computed both within- and across modality, i.e., 3Fa-(USa-
$\mathrm{USia}+\mathrm{UFa})$ for auditory; 3Fv-(USv+USiv+UFv) for visual; and the conjunction of these two for cross-modal.

The statistical threshold in all of the above analyses was set at $P<0.001$ for height, and corrected to $P<0.05$ for multiple comparisons (false discovery rate, FDR) at the cluster-level using cluster size. The exact values used as extent thresholds for particular contrasts are provided in the caption of Table II. For all the masks, $P<0.05$ was used without applying a correction for multiple comparisons. An anatomical toolbox-Anatomical Automatic Labeling (AAL) - was used to identify the activated structures [Tzourio-Mazoyer et al., 2002].

\section{RESULTS}

\section{Behavioral Data}

Table I presents correct responses (in percentage terms) and mean RTs during the familiar vs. unfamiliar discrimination task. No significant differences in the accuracy rate were found between conditions.

ANOVA on RTs revealed the significant main effect of modality $\left(F_{1,20}=7.81 ; P=0.011\right)$, type of name $\left(F_{5,100}=\right.$ 5.88; $P=0.001)$ and the modality $\times$ type of name interaction $\left(F_{5,100}=3.5 ; P=0.013\right)$. Because the interaction was significant we did not further test the main effects. Post hoc analysis of this interaction revealed that Sv, Siv, USv, and USiv were recognized faster than Sa, Sia, USa, and USia, respectively. In the case of the famous and UF names, the between-modality difference was not significant. Moreover, in the visual session, subjects responded quicker to Sv than to USv and UFv names. No such differences were present in the auditory part of the experiment.

\section{fMRI Data}

Direct comparison of "self" and "significant other" conditions did not reveal any significant cross-modal or modality-specific activations. Table II presents the pattern of activations associated with the processing of personally relevant names. Cross-modal activations were found specifically in the MPFC (Fig. 1A). Table II and Figure 1B illustrate brain activations for personally relevant names 
TABLE II. Cross-modal and modality-specific activations for self-name and for personally relevant names

\begin{tabular}{|c|c|c|c|c|}
\hline & $T$-value & $x$ & $y$ & $z$ \\
\hline \multicolumn{5}{|l|}{$\begin{array}{l}\text { Activations specific for self-name: } \\
\text { Cross-modal (Sa-Sia conj. Sv-Siv) }\end{array}$} \\
\hline No signifcant activation & - & - & - & - \\
\hline \multicolumn{5}{|c|}{ Auditory-specific [(Sa-Sia)-(Sv-Siv) masked incl. Sa-Sia] } \\
\hline No signifcant activation & - & - & - & - \\
\hline \multicolumn{5}{|c|}{ Visually-specific [(Sv-Siv)-(Sa-Sia) masked incl. Sv-Siv] } \\
\hline No signifcant activation & - & - & - & - \\
\hline \multicolumn{5}{|c|}{ Activations specific for personally relevant names: } \\
\hline \multicolumn{5}{|c|}{ Cross-modal (Sa-Fa conj. Sia-Fa conj. Sv-Fv conj. Siv-Fv)* } \\
\hline Medial prefrontal cortex & 3.71 & 4 & 56 & 26 \\
\hline \multicolumn{5}{|c|}{ Auditory-specific (Sa-Fa)-(Sv-Fv) conj. (Sia-Fa)-(Siv-Fv)** } \\
\hline Left Inferior Frontal Gyrus & 4.14 & -32 & 28 & -4 \\
\hline Right Inferior Frontal Gyrus & 4.05 & 50 & 20 & 10 \\
\hline \multicolumn{5}{|c|}{ Visually-specific (Sv-Fv)-(Sa-Fa) conj. (Siv-Fv)-(Sia-Fa) } \\
\hline No signifcant activation & - & - & - & - \\
\hline
\end{tabular}

Coordinates (in MNI space) and T-values of the peak activations which surpassed the $P<0.05$ level (correction for multiple comparisons). S, self-name; Si, significant other's name; F, famous person's name; a, auditory presentations; $v$, visual presentations.

*extent threshold at $k \geq 191$ voxels.

**extent threshold at $k \geq 135$ voxels.

that were specific for the auditory domain. Two brain structures were identified in this contrast: the left and right IFG. An analogous comparison for the visual modality failed to reveal any significant activations. Moreover, we did not find any significant activations specific for the general familiarity factor. As a consequence, there were no overlapping activations for self-specificity/personal-relevance and general familiarity.

\section{DISCUSSION}

This study is the first to compare auditory and visual recognition of self-, significant other, famous, and unknown names on the same group of subjects and with the same stimuli. The two main aims of this study were (1) to identify brain regions involved in self-name processing that are common and/or specific for the two modalities, and (2) to identify brain regions involved in the processing of personally relevant names (i.e., self- and significant other's name) which are common and/or specific for the two modalities. In addition, we tested whether activations for self-specificity/personal-relevance overlap with activations specific for the general familiarity factor.

Analysis of the behavioral data showed that following visual presentation of the stimuli, RTs to self-name were shorter than to USv and UFv names. This suggests that the former grabbed the participant's attention to a greater extent than the latter [Tacikowski and Nowicka, 2010], which consequently might have led to the faster recognition of this stimulus. On the other hand, the recognition of USv and UFv names could have been associated with some active inhibition processes that were not engaged in the case of self-name, i.e., "at first glance" USv and UFv may have appeared to the participants as self- or famous names and overcoming this initial response could have taken additional time. Alternatively, this difference might be explained by the task difficulty, since correct recognition of a highly familiar stimulus (i.e., self-name) seems to require less effort/time than the recognition of unknown names.

It is noteworthy that no differences in RT were found following auditory presentation of the stimuli. The analysis of RTs also revealed that some names were recognized faster when presented visually than aurally. This effect seems to be related to the slight difference in the duration of the visual vs. auditory stimuli (see the Methods section) and the stimuli presentation-in the case of auditory stimuli, the subjects had to wait to hear the family name to correctly identify the name holder, whereas with visual presentation, the complete information necessary for correct recognition was available immediately.

With regard to the first aim of this study, the self- vs. significant other's name contrast did not reveal any significant activations that were either modality-specific or crossmodal. The negative result is consistent with the study of Sugiura et al. [2008a], who also failed to detect any significant effects in the self- vs. friend's name contrast. These findings suggest that when names used as control conditions are adjusted to the self-name in respect of their emotional value and/or the frequency of occurrence and/or the personal-relevance, the difference between self- and other-name processing is reduced or even eliminated. A similar pattern has been observed in behavioral studies of the self-reference effect: the superiority in recall or recognition performance for the material which refers to "self" was shown to depend on the degree of intimacy between the participant and the person used for comparison [for review see Symons and Johnson, 1997]. 


\section{A. Cross-modal activations for personally-relevant names}
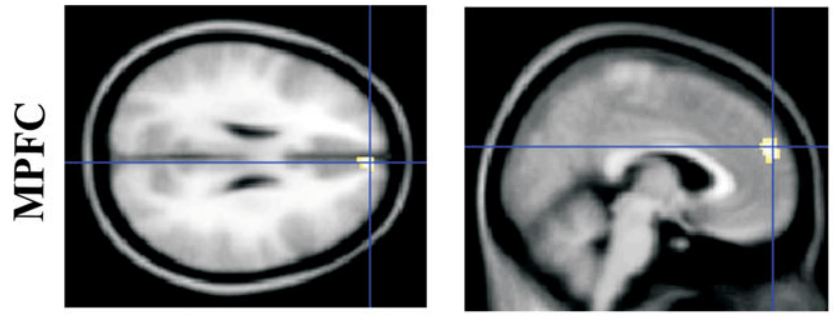

\section{B. Auditory-specific activations} for personally-relevant names
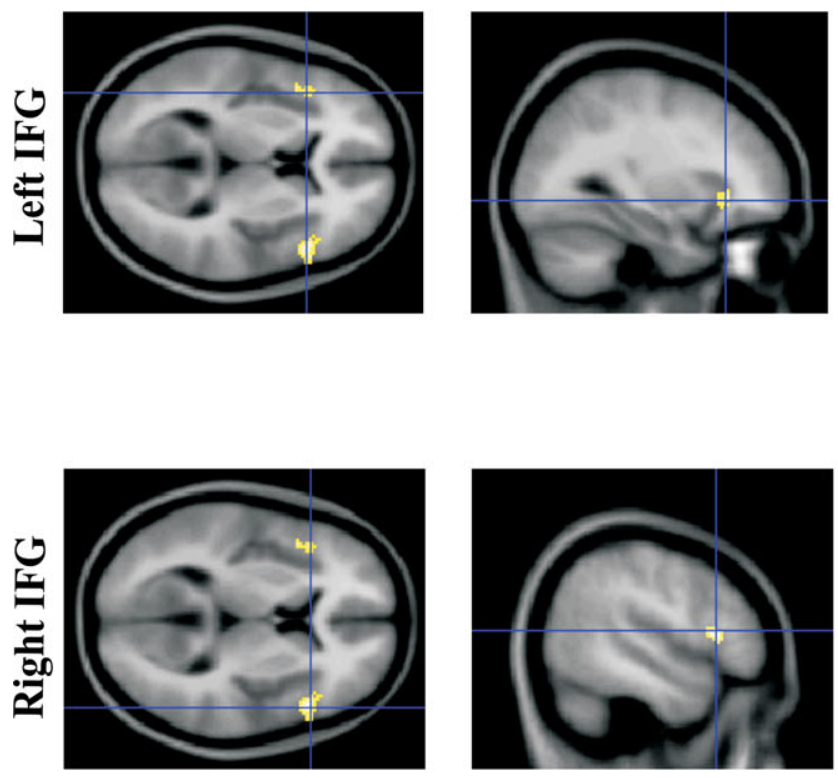
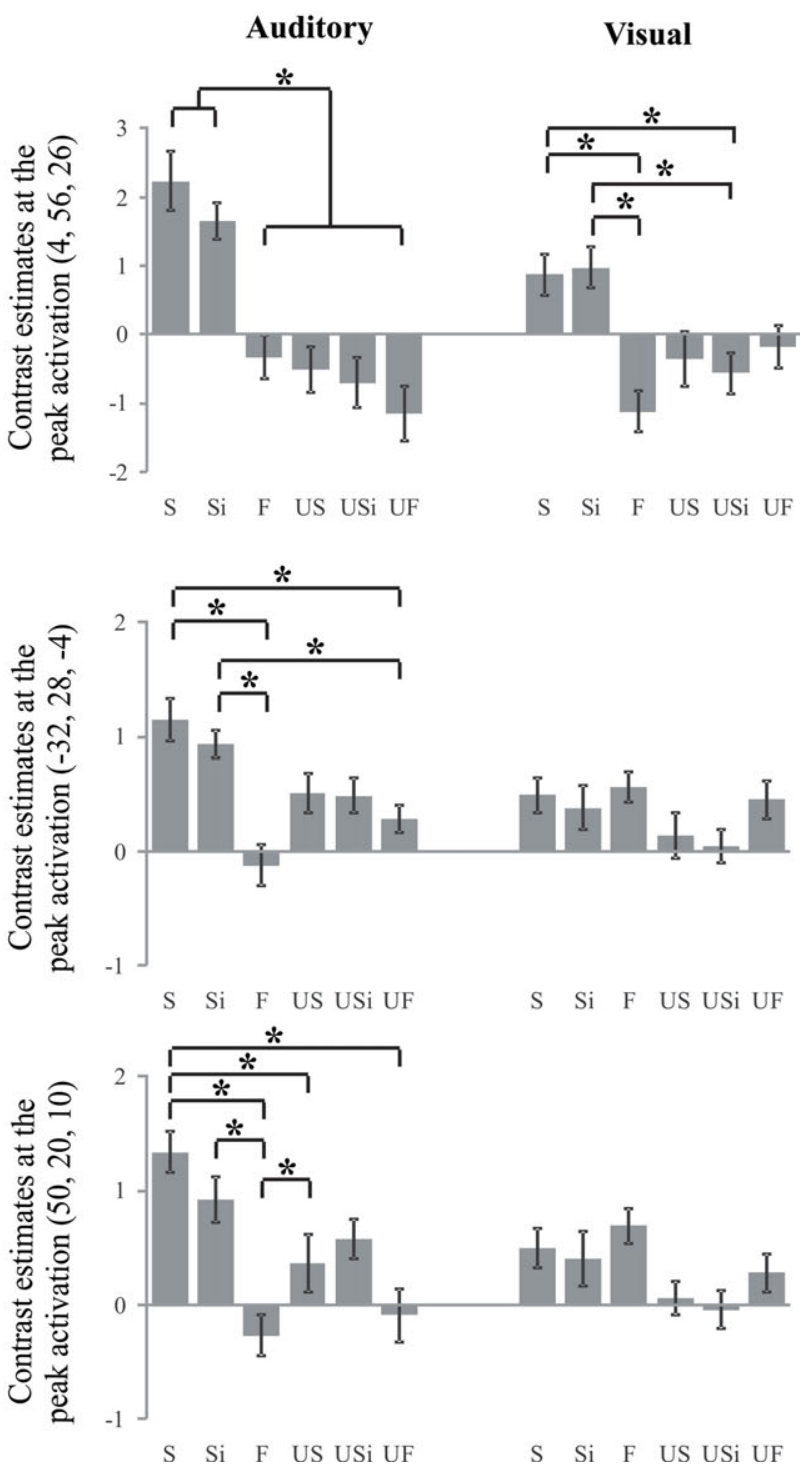

Figure I.

Cross-modal activations common for self- and significant other's name were found specifically in the medial prefrontal cortex (A). Auditory-specific activations common for these two names were found in the left and right inferior frontal gyri (B). The activation map is superimposed on a template of 152 averaged TI images (MNI). The activation maps are corrected for multiple comparisons at the cluster level. Bar graphs show contrast estimates at the peak activations (error bars represent standard

As far as the second aim of this study is concerned, we tested both cross-modal and modality-specific activations for personally relevant names. Personal relevance was operationalized as a common response to self- and significant other's name vs. a famous name. Cross-modal effects were found in the MPFC. The involvement of the MPFC in the processing of personally relevant names is consist- error of the mean). These data were examined post hoc using differences are marked with *. S, self-name; Si, significant other's name; F, famous person's name; US, unknown name with the same first name as the subject; USi, unknown name with the same first name as the significant other; UF, unknown name with the same first name as the famous person; a, auditory modality; v, visual modality.

ent with the results of previous studies [e.g., Kampe et al., 2003; Perrin et al., 2005; Staffen et al., 2006]. This study, together with that of Sugiura et al. [2008a], extends the previous findings by showing that the level of activation in the MPFC is similar in the case of self- and close other's name processing. This pattern of results suggests that this structure does not play a role in self-other distinction, but paired $t$-tests (two-tailed). The significant (Bonferroni corrected) 
rather in the processing of personally relevant stimuli [Sugiura et al., 2008a].

The MPFC involvement in the recognition of personally relevant names, regardless of their modality, seems to be in line with the findings of studies on self-referential processing, i.e., when subjects decide how well a trait adjective describes them [Heatherton et al., 2006; Johnson et al., 2002; Kelley et al., 2002; Mitchell et al., 2006; Seger et al., 2004; Vanderwal et al., 2008, Yaoi et al., 2009]. Activations in the cortical midline structures, including the MPFC, have also been recorded during self-reflection tasks where participants are asked to think about their hopes and aspirations or their duties and obligations [Johnson et al., 2006]. Activity in the MPFC was also observed in tasks in which participants made judgments about others, especially "close others" [Ochsner et al., 2005], or about people they see as similar to themselves [Mitchell et al., 2006]. On the basis of this evidence, it has been suggested that the MPFC is engaged in the processing of information related to "self" [see Northoff et al., 2006, for a meta-analysis] and our results are consistent with this hypothesis.

The cross-modal MPFC engagement in the processing of personally relevant names could also be interpreted in terms of involvement of the "default-mode network" which includes the MPFC [e.g., Gusnard et al., 2001; Raichle et al., 2001; for a review]. This network shows consistent decreases in activity when subjects perform external, goal-directed tasks. On the basis of these findings it was proposed that the "default mode network" is engaged in internally driven processing. It has also been observed that these task-related deactivations overlap considerably with task-related activations during social cognition tasks, i.e. the "theory of mind" and "mentalizing" [Schilbach et al., 2008]. This overlap could be related to the fact that a large amount of internally driven processing is probably social in nature. Subjects left alone in the scanner, with their eyes closed, might engage in a series of social-related processes like "What does person A think about something?", "What do I think about it?" etc. [Schilbach et al. 2008]. Developmental psychology suggests that self-consciousness emerges from comparing own cognitive states with those of others; therefore, internally driven processing and social cognition might in fact engage similar psychological and neural processes [Schilbach et al., 2008]. In this context, the increased activation of MPFC for self- and significant other's name could be explained by the involvement of the "default mode network" in response to internally/socially relevant stimuli.

Auditory-specific activations for personally relevant names (self- and significant other's name) were found in the left and right IFG. Involvement of the bilateral IFG was also identified in previous studies on auditory selfname processing [Holeckova et al., 2008; Kampe et al., 2003], but not in studies which presented names visually [Sugiura et al., 2006, 2008a,b]. On the other hand, in the experiment of Kaplan et al. [2008], the right IFG was the only structure that showed cross-modal activation for self- face and self-voice. It should be mentioned that activation of these regions has also been demonstrated in a variety of other tasks, e.g., auditory word recognition [e.g., see Blumstein, 2009, for a review), self-face recognition [see Platek et al., 2008, for a meta-analysis], or inhibition processes [see Aron et al., 2004, for a review). The fact that bilateral IFG also showed increased responses to USa and USia names in our study (see the bar graphs in Fig. 1) suggests that these regions might be involved in some automatic switch of attention towards personally relevant names. In the auditory modality, the beginning (the first name) of $\mathrm{Sa} / \mathrm{Sia}$ and USa/USia were the same; therefore, USa/USia could have activated the same automatic attention processes as Sa and Sia, respectively (cf. the "cocktail party" phenomenon, e.g. Moray [1959]). Besides this interpretation, the reason why we did not observe any effects in IFG in the visual domain might be due to their smaller adaptational value, i.e., in most everyday life situations fast and adequate reaction to hearing a name seems more crucial than to seeing it.

The above interpretation seems to fit well with the hypothesis of Corbetta and Shulman [2002]. They proposed two partially segregated brain networks engaged in different attentional functions. The dorsal frontoparietal network is involved in preparation and application of goaldirected selection of stimuli and responses (top-down processing), whereas the ventral frontoparietal network is involved in the detection of behaviorally relevant stimuli, especially when they are salient or unexpected (bottom-up processing). In this model, the IFG are part of the ventral frontoparietal network; therefore, the increased activation that we observed could indicate the engagement of bottom-up processes triggered by personally relevant names. The authors state that "Although, in real life, there is no question that sudden unfamiliar stimuli can grab our attention no matter what we are thinking at the moment, it is also possible that some stimuli attract attention because of some form of contingency that is hard-wired in the brain by learning, development or genetics." (p. 208). Increased activation in the IFG for personally relevant names seems to be consistent with this view.

So far, we have focused on how personally relevant information is processed in the brain. However, it is also important to ask why such information is processed preferentially. This preference could be attributed either to the high social/adaptive value of personally relevant stimuli (their subjective importance) or the high familiarity of these stimuli (one's own name and that of a significant other are probably the most frequently heard and used names in everyday life). On the basis of this assumption, it might be reasoned that if general familiarity was the only factor responsible for the effects that we observed, then the difference between famous and unknown names (famous $>$ unknown) should have coincided with the difference between personally relevant and famous names (personally relevant $>$ famous). However, the results of famous $>$ unknown contrasts did not overlap with 
personally relevant activations. In fact, these contrasts did not reveal any significant activations.

This pattern of results is consistent with those of Qin et al. [2012] and emphasizes that the preferential processing of personally relevant stimuli cannot be explained just by general familiarity. On the other hand, the unknown names that we used in this study could be atypical: because they had the same beginnings as the subject's, the close other's and the famous person's names, their processing may have been different from that of typical unknown names. It is also noteworthy that we did not control the familiarity of names within the "famous" category, i.e., although we tried to select names of only very well-known individuals, e.g., "Steffi Graf, Boris Becker" etc., some of the names could have been more familiar than the other. This variability could explain the lack of differences in processing famous and unknown names in this study. Further research is needed to differentiate effects of personal-relevance and general familiarity.

Another possibility that could not be ruled out is that behavioral results (shorter RTs) could have influenced the activation pattern observed in this study. However, if that was the case, some consistency between these two should be expected. The opposite was found: (1) effects in MPFC were present for both modalities, whereas differences in RTs were present only in the visual domain; and (2) effects in IFG were observed for auditory stimuli only, for which no RTs effects were found. On the basis of the above considerations, the reported neural activations does not seem to be a mere reflection of the behavioral effects.

In conclusion, the novel contribution of this study is the identification of modality-specific and cross-modal patterns of neural responses to personally relevant names. Our findings suggest that separate brain structures might be engaged in different aspects of reacting to personally relevant names: activation in the MPFC could reflect selfreferential processes, while bilateral IFG activation, present specifically in the auditory domain, might be associated with the automatic switch of attention toward personally relevant names.

\section{ACKNOWLEDGMENTS}

The authors thank Monika Dobrowolny for technical assistance.

\section{REFERENCES}

Aron AR, Robbins TW, Poldrack RA (2004): Inhibition and the right inferior frontal cortex. Trends Cogn Sci 8:170-177.

Baron-Cohen S, Allen J, Gillberg C (1992): Can autism be detected at 18 months? The needle, the haystack, and the CHAT. Br J Psychiatry 161:839-843.

Blumstein SE (2009): Auditory word recognition: Evidence from aphasia and functional neuroimaging. Lang Linguist Compass 3:824-838

Carmody DP, Lewis M (2006): Brain activation when hearing one's own and others' names. Brain Res 1116:153-158.
Cherry EC (1953): Some experiments on the recognition of speech, with one and with two ears. J Acoust Soc Am 25:975-979.

Corbetta M, Shulman GL (2002): Control of goal-directed and stimulus-driven attention in the brain. Nat Rev Neurosci 3:201-215.

Friston KJ, Holmes AP, Worsley KJ, Poline JP, Frith CD, Frackowiak RSJ (1995): Statistical parametric maps in functional imaging: A general linear approach. Hum Brain Mapp 2:189-210.

Greenhouse S, Geisser S (1959): On methods in the analysis of profile data. Psychometrica 24:95-112.

Gronau N, Cohen A, Ben-Shakhar G (2003): Dissociations of personally significant and task-relevant distracters inside and outside the focus of attention: A combined behavioural and psychophysiological study. J Exp Psychol 132:512-529.

Gusnard DA, Raichle ME (2001): Searching for a baseline: Functional imaging and the resting human brain. Nat Rev Neurosci 2:685-694.

Heatherton TF, Wyland CL, Macrae CN, Demos KE, Denny BT, Kelley WM (2006): Medial prefrontal activity differentiates self from close others. Soc Cogn Affect Neurosci 1:18-25.

Holeckova I, Fischer C, Morlet D, Delpuech C, Costes N, Mauguiere F (2008): Subject's own name as a novel in a MMN design: A combined ERP and PET study. Brain Res 1189:152-165.

Johnson SC, Baxter LC, Wilder AS, Pipe JG, Heiserman JE, Prigatano GP (2002): Neural correlates of self-reflection. Brain 125:1808-1814

Johnson MK, Raye CL, Mitchell KJ, Touryan SR, Greene EJ, Nolen-Hoeksema S (2006): Dissociating medial frontal and posterior cingulate activity during self-reflection. Soc Cogn Affect Neurosci 1:56-64

Kampe KK, Frith CD, Frith U (2003): Hey John: signals conveying communicative intention toward the self active brain regions associated with mentalizing, regardless of modality. J Neurosci 23:5258-5263.

Kaplan JT, Aziaz-Zadeh L, Uddin LQ, Iacoboni M (2008): The self cross the senses: An fMRI study of self-face and self-voice recognition. Soc Cogn Affect Neurosci 3:218-223.

Kelley WM, Macrea CN, Wyland CL, Caglar S, Inati S, Heatherton TF (2002): Finding the self? An event-related fMRI study. J Cogn Neurosci 14:785-794.

Koole SL, Pelham BW (2003): On the nature of implicit selfesteem: The case of the name letter effect. In: Spencer SJ, Fein S, Zanna MP, Olson JM, editors. Motivated Social Perception: The Ontario Symposium. Hillsdale, NJ: Lawrence Erlbaum. pp93-116.

Laureys S, Perrin F, Brédart S (2007): Self-consciousness in noncommunicative patients. Conscious Cogn 16:722-741.

Mitchell JP, Macrae CN, Banaji MR (2006): Dissociable medial prefrontal contributions to judgments of similar and dissimilar others. Neuron 50:655-663.

Moray N (1959): Attention in dichotic listening: Affective cues and the influence of instructions. Q J Exp Psychol 11:56-60.

Northoff G, Heizel A, de Greck M, Bermpohl F, Dobrowolny H, Panksepp J (2006): Self-referential processing in our brain: A meta-analysis of imaging studies on the self. NeuroImage 31:440-457.

Ochsner KN, Beer JS, Robertson ER, Cooper JC, Gabrieli JDE, Kihsltrom JF, D'Eposito M (2005): The neural correlates of direct and reflected self-knowledge. Neuroimage 28:797-814.

Parise E, Friederici AD, Striano T (2010): “Did you call me?" 5month-old infants own name guides their attention. PLoS One 5:e14208. 
Perrin F, Maquet P, Peigneux P, Ruby P, Degueldre C, Balteau E, Del Fiore G, Moonen G, Luxen A, Laureys S (2005): Neural mechanisms involved in the detection of our first name: A combined ERPs and PET study. Neuropsychologia 43:12-19.

Platek SM, Wathne K, Tierney NG, Thomson JW (2008): Neural correlates of self-face recognition: An effect-location meta-analysis. Brain Res 1232:173-184.

Qin P, Liu Y, Shi J, Wang Y, Duncan N, Gong Q, Weng X, Northoff $G$ (2012): Dissociation between anterior and posterior cortical regions during self-specificity and familiarity: A combined fMRI-meta-analytic study. Hum Brain Mapp 33:154-164.

Raichle M, MacLeod AM, Snyder AZ, Powers WJ, Gusnard D, Schulman GL (2001): A default mode of brain function. Proc Natl Acad Sci USA 98:676-682.

Schilbach L, Eickhoff SB, Rotarska-Jagiela A, Fink GR, Vogeley K (2008): Mind at rest? Social cognition as the default mode of cognizing and its putative relationship to the "default system" of the brain. Conscious Cogn 17:457-467.

Seger CA, Stone M, Keenan JP (2004): Cortical activations during judgments about the self and another person. Neuropsychologia 42:1168-1177.

Shapiro KL, Caldwell J, Sorensen RE (1997): Personal names and the attentional blink: A visual cocktail party effect. J Exp Psychol Hum Percept Perform 23:504-514.

Staffen W, Kronbichler M, Aichhorn M, Mair A, Ladurner AM (2006): Selective brain activity in response to one's own name in the persistent vegetative state. J Neurol Neurosurg Psychiatry 77:1383-1384.

Sugiura M, Sassa Y, Hyeonjeong J, Miura N, Akitsuki Y, Horie K, Sato S, Kawashima R (2006): Multiple brain network for visual self-recognition with different sensitivity for motion and body part. NeuroImage 32:1905-1917.
Sugiura M, Sassa Y, Jeong H, Horie K, Sato S, Kawashima R (2008a): Face-specific and domain-general characteristics of cortical responses during self-recognition. NeuroImage 42:414-422.

Sugiura M, Sassa Y, Watanabe J, Akitsuki Y, Maeda Y, Matsue Y, Kawashima R (2008b): Anatomical segregation of representations of personally familiar and famous people in the temporal and parietal cortices. J Cogn Neurosci 21:1855-1868.

Symons CS, Johnson BT (1997): The self-reference effect in memory: A meta-analysis. Psychol Bull 121:371-394.

Tacikowski P, Nowicka A (2010): Allocation of attention to selfname and self-face: An ERP study. Biol Psychol 84:318-324.

Tacikowski P, Brechmann A, Marchewka A, Jednoróg K, Dobrowolny M, Nowicka A (2011): Is it about the self or the significance? An fMRI study of self-name recognition. Soc Neurosci 6:98-107.

Tzourio-Mazoyer N, Landeau B, Papathanassiou D, Crivello F, Etard O, Delcroix N, Mazoyer B, Joliot M (2002): Automated anatomical labelling of activations in spm using a macroscopic anatomical parcellation of the MNI MRI single subject brain. Neuroimage 15:273-289.

Vanderwal T, Hunyadi E, Grupe DW, Connors CM, Schultz RT (2008): Self, mother and abstract other: An fMRI study of reflecting social processing. Neuroimage 41:1437-1446.

Wager TD, Nichols TE (2003): Optimization of experimental design in fMRI: A general framework using a genetic algorithm. NeuroImage 18:293-309.

Wentura D, Kulfalnek M, Greve W (2001): Masked affective priming by own-name letters: Evidence for a correspondence between explicit and implicit self-esteem. J Exp Soc Psychol 41:654-663.

Yaoi K, Osaka N, Osaka M (2009): Is self special in the dorsomedial prefrontal cortex? An fMRI study. Soc Neurosci 4:455-463. 